\title{
Relevamiento sociodemográfico del aprovechamiento de la fauna íctica en la cuenca inferior del río Luján
}

\author{
Paula Courtalon ${ }^{1}$, Gabriela Gerardo ${ }^{1}$ y Liliana Ciotek $^{2}$
}

${ }^{1}$ Grupo de Investigación en Ecología de Humedales. Facultad de Ciencias Exactas y Naturales. Universidad de Buenos Aires. Argentina. ${ }^{2}$ Parque Nacional Campos del Tuyú. Administración de Parques Nacionales. Argentina.

E-mail: pcourtalon@gmail.com

RESUMEN. El objetivo general del presente trabajo fue identificar las especies de peces que capturan los pescadores y el uso que le dan a las mismas en la cuenca inferior del río Luján. Para tal fin, se realizaron entrevistas semiestructuradas durante la primavera de 2015, el verano y el otoño 2016, las cuales permitieron obtener información sobre el uso que les dan los pescadores a los peces capturados. Se analizaron a lo largo de este período dos escenarios: los días de semana y los fines de semana. En la cuenca inferior del río Luján coexiste una diversidad de contextos sociales manifestados a través de sus actores: los pobladores locales y los pobladores de partidos vecinos que visitan el área. Las especies más mencionadas a lo largo de todo el estudio fueron: Pimelodus maculatus, Pimelodus albicans, Astyanax sp., Megaleporinus obtusidens y Rhamdia quelen. Los resultados obtenidos permiten plantear la importancia que tiene este territorio en la ribera del río Luján para los pobladores de la zona y de las regiones cercanas dado que los días de semana es un sitio que ofrece un espacio para realizar pesca de subsistencia mientras que los fines de semana constituye un espacio para el esparcimiento familiar.

Palabras clave: Entrevistas, Otamendi, pesca de subsistencia, relevamiento sociodemográfico, río Luján.

ABSTRACT. The general objective of the present work was to identify the fish species that the fishermen capture and the use they give to them at the lower basin of the Luján River. To this purpose, semi-structured interviews were conducted, which allowed obtaining information on the use that fishers give to the captured fish during the spring of 2015, summer and autumn 2016. Throughout this period, two scenarios were analyzed: weekdays and weekends. At the lower basin of the Luján River, a diversity of social contexts manifested through its actors coexists: the local inhabitants and the inhabitants of neighboring municipalities that visit the area. The most mentioned species throughout the study were: Pimelodus maculatus, Pimelodus albicans, Astyanax sp., Megaleporinus obtusidens y Rhamdia quelen. The obtained results revealed the importance of this territory situated at banks of the Luján River for the inhabitants of the area and the nearby regions due to it is a location that on weekdays offers a space for subsistence fishing, and on weekends for family recreation.

Keywords: Interview, Luján River, Otamendi, sociodemographic survey, subsistence fishing.

\section{INTRODUCCIÓN}

En la porción continental e insular del Bajo Delta del río Paraná (Kandus et al., 2006), en el Noreste de la provincia de Buenos Aires, puede observarse que coexiste una diversidad de contextos sociales manifestados a través de sus actores: los pobladores locales y los pobladores de partidos vecinos que visitan el área de estudio. 
En el Barrio Estación río Luján y en la zona aledaña al barrio Otamendi (zona continental del Partido de Campana), es habitual que los pobladores utilicen el área para la pesca. De entrevistas realizadas a pescadores en la zona de río Luján se desprende que pescan fundamentalmente bagres blancos, Pimelodus albicans (Valenciennes, 1840) y amarillos, Pimelodus maculatus Lacepède, 1803, mojarras (Astyanax sp.) y anguilas, Synbranchus marmoratus Bloch, 1795 y de las entrevistas sobre uso de fauna realizadas a pobladores locales de los barrios de Otamendi y de río Luján también se obtuvo como resultado que se realiza la caza de subsistencia de coipos, Myocastor coypus (Molina, 1782), carpinchos, Hydrochoerus hydrochaeris, (Linnaeus, 1766), liebres, Lepus europaeus (Pallas, 1778), ranas criollas, Leptodactylus latrans (Steffen, 1815) y pájaros (Cruz y Courtalon, 2017).

Fassel (1997) expresa que hacia la mitad del siglo $X X$, en el Molino viejo (ubicado en la margen opuesta al área de estudio en el río Luján) había bogas, Megaleporinus obtusidens (Valenciennes, 1836 ), patíes, Luciopimelodus pati (Valenciennes, 1836), doradillos, Salminus brasiliensis (Cuvier, 1816), tarariras, Hoplias argentinensis Rosso, González-Castro, Bogan, Cardoso, Mabragaña, Delpiani, y Díaz de Astarloa, 2018, surubíes pintado Pseudoplatystoma corruscans (Spix y Agassiz, 1829), bagres, mojarras (Astyanax sp.), dientudos, Oligosarcus jenynsii (Günther, 1964) y sábalos, Prochilodus lineatus (Valenciennes, 1836), de gran tamaño. Liotta et al. (2003) plantean que a partir de 1980 debido al importante grado de deterioro de las variables limnológicas se comienza a observar que la mayoría de las especies anteriormente mencionadas empezó a desaparecer. Durante los años 2014 a 2016 y como parte del relevamiento ictiológico realizado en la Reserva Natural Otamendi (RNO) y zonas aledañas (Ciotek et al., 2017) muchas de las especies observadas en el siglo pasado se volvieron a encontrar, registrándose ejemplares de tamaño pequeño o mediano.

En la entrevista semiestructurada el entrevistador crea con anticipación las preguntas, pero posee la libertad de alterar el orden de éstas, sin olvidar los objetivos de la investigación.
Este tipo de entrevista busca construir un cuadro acerca de uno o varios puntos de interés (Lucca Irizarry y Berríos Rivera, 2009). Permite documentar el conocimiento ecológico tradicional, dirigir las discusiones en la medida necesaria para cubrir temas específicos más a fondo, discutir y describir puntos de vista de los temas, y establecer conexiones basadas en dichos puntos de vista más allá de las preguntas redactadas de antemano por el investigador (Abba \& Casini, 2010).

Las percepciones pueden ser dilucidadas a través de las referencias al uso, ya que gracias a nuestros patrones culturales estructuramos nuestros conocimientos y percepciones de forma individual y colectiva, lo que determinará los cursos de acción y las decisiones tomadas hacia la fauna (Navarro, 2005). Identificar las percepciones acerca de la fauna puede producir información útil para ser incorporada en la toma de decisiones de conservación y en la resolución de conflictos entre los pobladores locales y las autoridades (Vodouhê et al., 2010).

Para asignar un uso cualitativo a las especies mencionadas se consideraron categorías establecidas de uso (Monroy-Vilchis et al., 2008; Tejada et al., 2006). El objetivo general del presente trabajo fue identificar las especies de peces que capturan los pescadores y el uso que le dan a las mismas considerando tres escenarios posibles: A) Las especies capturadas a lo largo de todo el período de estudio (primavera 2015, verano y otoño 2016), B) Las especies capturadas los días de semana en el período de estudio y C) Las especies capturadas los fines de semana en el período de estudio. Además, se proponen los siguientes objetivos particulares: en primer término, la identificación de qué especies se capturan y posteriormente para cada uno de los escenarios mencionados qué especies se capturan en cada uno, la localidad de origen del pescador, el arte de pesca utilizado, tipo de carnada, si todos los peces capturados fueron consumidos y el período del año en que realiza la actividad.

\section{MATERIALES Y MÉTODOS Área de estudio}

En sentido ictiogeográfico el sitio de muestreo se 
encuentra en la ecorregión de agua dulce Lower Paraná (Hales \& Petry, 2015) (Figura 1), un área de considerable riqueza ictícola. Esta ecorregión
(45\%) y Siluriformes (31\%), en menor proporción los Cyprinodontiformes (7\%), Perciformes (6\%), Gymnotiformes (3\%), Myliobatiformes,

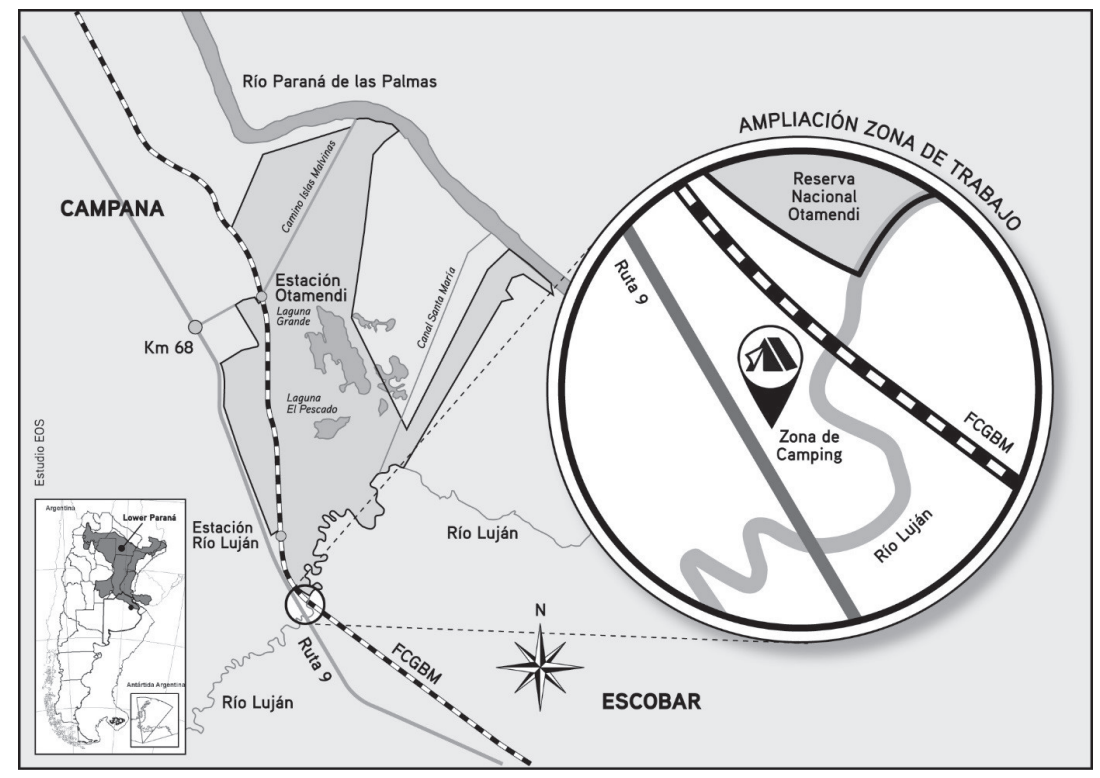

Figura 1. Ubicación del área de estudio. En gris oscuro los límites de la Reserva Natural Otamendi. Fuente: Estudio de Diseño EOS.

Figure 1. Location of the study area. In dark gray the limits of the Otamendi Nature Reserve. Source: EOS Design Studio.

comprende la parte inferior del drenaje del río Paraná aguas abajo de las cataratas de Guaíra (Salto de Sete Quedas) y la presa de Itaipú, una pequeña porción del río Paraguay aguas abajo de la confluencia del río Bermejo, la parte inferior del río Iguazú debajo de las Cataratas del Iguazú, así como diversos cursos de agua que desembocan directamente en el Río de la Plata. El número de especies presentes en la ecorregión Lower Paraná disminuye hacia el sur, principalmente debido a cambios en la temperatura (Bonetto et al., 1986). Entre los peces más notables se encuentran el sábalo ( $P$. lineatus) que constituye más del $50 \%$ de la biomasa de peces en el Paraná inferior (Bonetto et al., 1986), así como bogas (M. obtusidens) y dorados (S. brasiliensis) que son especies que realizan importantes migraciones para reproducirse.

Ciotek et al. (2017) citan para la RNO, cuyo límite sur coincide con el sitio de muestreo de este trabajo, 102 especies de peces, siendo los órdenes con mayor riqueza específica los Characiformes
Clupeiformes y Cypriniformes (2\%) y por último Atheriniformes y Synbranchiformes (1\%). La presencia de especies con escaso registro en otras áreas protegidas de la Administración de Parques Nacionales de la Argentina como las mojarras Hyphessobrycon reticulatus Ellis, 1911 y Mimagoniates inequalis (Eigenmann, 1911), aumentan el valor de la RNO como área de conservación y refuerzan la necesidad del cuidado del medio acuático.

La cuenca del río Luján se encuentra en la ecoregión Pampa y en la subregión denominada pampa ondulada (Viglizzo et al., 2005). Presenta una planicie inundable de suave pendiente hacia el Río de la Plata que ha sido modificada en parte con el relleno y la construcción de emprendimientos inmobiliarios, canalización, etc. Esto produce cambios en la dinámica hidrológica y altera las características ecológicas del humedal (Morello et al., 2000).

El río Luján tiene su nacimiento en el partido de Suipacha al NO de la Provincia de Buenos Aires $\left(59^{\circ} 37^{\prime}\right.$ W, $\left.34^{\circ} 43^{\prime} \mathrm{S}\right)$. Es un río de llanura que se 
origina a partir de la confluencia de los arroyos Los Leones y Durazno. Su cuenca posee una superficie de $3000 \mathrm{~km}^{2}$ y una longitud de 130 km (Andrade, 1986). La dirección del recorrido del río Luján, es SO-NE desde su inicio hasta la altura del cruce con la Ruta Nacional $N^{\circ} 9$ donde desarrolla una planicie aluvial meandrosa. Luego cambia el rumbo hacia la dirección SE paralelo al río Paraná de la Palmas para finalmente confluir en el Delta del río Paraná (partido de Tigre) y desemboca en el estuario del Río de la Plata (Figura 2). Aguas abajo del área de estudio, en el lugar donde el río cambia hidrológico está afectado por las fluctuaciones del río Paraná junto con las mareas y sudestadas del Río de la Plata (Andrade, 1986).

El cauce principal del río Lujan posee una variación suave de la pendiente desde su nacimiento hasta su desembocadura que permite dividir su curso en tres secciones (Brea, 2007): curso superior, intermedio e inferior. En el curso inferior (donde se ubica nuestra área de estudio) posee una longitud de $60 \mathrm{~km}$ con una pendiente de aproximadamente de 0,04 $\mathrm{m} / \mathrm{km}$ y es donde se realizan las actividades relacionadas con usos urbanos y recreativos.

\section{Cuenca del río Luján, Buenos Aires, Argentina}

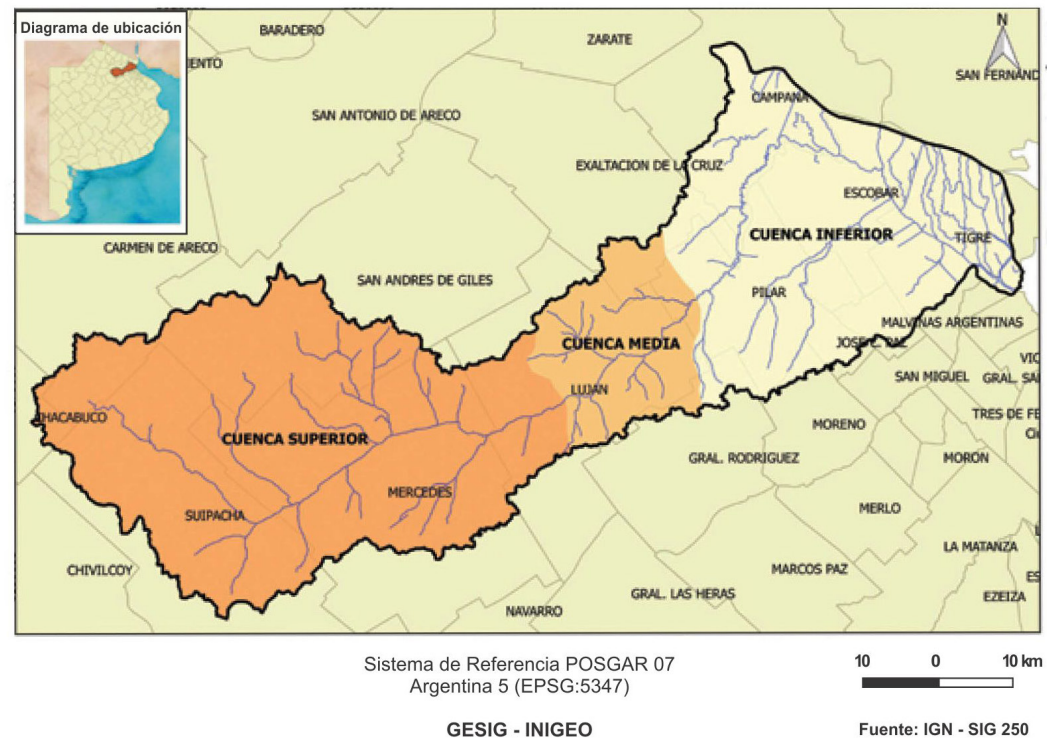

Figura 2. Cuenca hidrográfica del río Luján (Modificado de Buzai y Principi, 2017).

Figure 2. Luján river hydrographic basin (Modificated from Buzai y Principi, 2017).

de dirección, se conecta con el río Paraná de las Palmas a través del canal artificial Santa María (Figura 1).

La región de la cuenca del río Luján posee un clima templado húmedo con temperaturas medias mensuales de $16,6^{\circ} \mathrm{C}$. Las precipitaciones medias anuales son de $951 \mathrm{~mm}$ (Goldberg et al., 1995) con máximos de 1300 a 1400 mm (Andrade, 1986). El régimen hidrológico del río es del tipo pluvial y por escurrimiento subterráneo en los cursos medios y superior, posee un caudal medio de $5,4 \mathrm{~m}^{3} / \mathrm{s}$, alcanzando máximos de $400 \mathrm{~m}^{3} / \mathrm{s}$ en épocas de grandes precipitaciones. En el cauce inferior el régimen
En este tramo el río recibe aportes significativos de contaminantes domiciliarios e industriales directamente sobre su curso. También hay aportes por parte de sus tributarios siendo el más importante el proveniente del río Reconquista.

El sitio en donde se realizaron las encuestas está ubicado sobre la margen izquierda del río Luján y su intersección con la RN 9 (5852' S, 34 $17^{\prime}$ O), partido de Campana. Dicho punto localmente pertenece a la cuenca inferior del río Luján (Andrade, 1986; Plataroti, 2010) y se conoce como camping Río Luján. Se accede a él a través de una calle colectora asfaltada paralela 
a la RN 9 que finaliza en el río y que permite el libre acceso de todo tipo de vehículos (Figura 1).

\section{TOMA DE DATOS}

Encuestas semi-estructuradas: A cada entrevistado se le explicó con antelación que las entrevistas se realizaban en el marco de un proyecto de extensión de la Facultad de Ciencias Exactas y Naturales de la Universidad de Buenos Aires y que el proyecto trataba acerca del uso de fauna silvestre, en particular sobre los peces. Se

\section{DESCRIPCIÓN DE LAS VARIABLES}

A continuación, se presentan las variables que forman el cuerpo de la encuesta. Cada una de ellas será luego expresada como porcentaje de referencia, que se calcula como la cantidad de veces que cada variable es mencionada por los entrevistados, en función del total de entrevistados que respondieron para cada variable en cuestión.

Especies pescadas: Las especies enunciadas por los entrevistados fueron identificadas sobre la

Tabla 1. Modelo de encuesta utilizado para realizar las entrevistas.

\section{ENTREVISTA A PESCADORES}

Entrevista No. Fecha:

\section{DATOS SOBRE EL ENCUESTADO}

Localidad de origen

Sexo: $\quad F \quad M$

Edad:

Ocupación:

1. ¿Usted se dedica principalmente a pescar o lo hace ocasionalmente como complemento de otra actividad?

Actividad principal Actividad complementaria

2. ¿Usted vive en río Luján o viene a pescar desde otra localidad?

río Luján Otra localidad ¿De cuál?

3. ¿Qué pesca? Mostrar mini-guía para corroborar.

4. ¿Hay peces que ya no salen o que están en poca cantidad o que son de talla chica?

Peces que ya no salen Peces en poca cantidad Peces de talla

chica ¿Cuáles?

5. ¿Todo lo que pesca lo consume? Si no ¿qué hace con los peces?

Consume todo ¿tro uso ¿Cuál?

6. ¿Con qué pesca?

Caña Medio Mundo Espinel/línea Red Otro

7. ¿Qué utiliza como carnada? La carnada, ¿la compra o la pesca en el mismo lugar?

Carnada: Comprada Pescada

8. ¿En qué época del año sale a pescar? ¿Durante cuántos días?

Época: Días

pidió su consentimiento y se le dio la opción de responder de forma anónima (Tabla 1). Todas las entrevistas se realizaron de forma presencial intentando establecer una conversación fluida con los entrevistados. base de fotos de las especies presentes en la zona de la Guía de los Peces del Parque Nacional Pre-Delta (Almirón et al., 2015).

Consumo de peces: Para asignar un uso cualitativo a las especies mencionadas, se 
consideraron categorías establecidas de uso alimento, deportivo u otros (carnada o alimento de mascota)-, (Monroy-Vilchis et al., 2008; Tejada et al., 2006). Además de en qué época del año se pesca, se relevaron también datos sociodemográficos tales como edad, localidad donde habitan y ocupación.

Las especies consumidas se expresaron como el porcentaje de referencia de consumo. En caso de que la respuesta fuera negativa se indagó si hubo pesca deportiva o no (otros usos: venta eventual, alimento de mascotas y carnada para la pesca). Se entiende por pesca deportiva que se captura el ejemplar y con el menor daño posible se lo devuelve al agua.

Arte de pesca utilizado: Según sus características operativas los diferentes artes de pesca normalmente utilizados en aguas continentales pueden ser agrupados en pasivos y activos (Padín e Iriart, 1991). Las artes pasivas son las se calan en un punto determinado y es el pez con su actividad y desplazamiento el que determina la captura. Las artes activas son las que al ser desplazadas filtran un volumen de agua que varía en función de la distancia recorrida y capturan los peces presentes en el área barrida, independientemente de su nivel de actividad. En el área de estudio se detectó el uso de los siguientes artes de pesca pasivos: a) Caña, consiste en una caña rígida con una línea fuerte al extremo anterior de ésta, la cual está equipada con un anzuelo. La línea es de nylon. Las cañas pueden ser de distintos materiales y tamaños. Se le pueden poner lineadas de flote o de fondo con tanzas de diferentes grosores y tamaños de boya, plomadas y anzuelos. Una caña con reel (bobina o carrete que contiene la tanza enrollada) permite que se amplíe el rango de distancia a la que se arroja la lineada y ayuda en la captura y colecta del pez; b) Línea, consiste en un carrete dónde se enrolla una línea de monofilamento al extremo de la cual se sujeta uno o varios anzuelos. En ocasiones se coloca un peso para que el anzuelo se hunda; c) Espinel, es en una cuerda a la cual se unen, a intervalos regulares, líneas con anzuelos; d) Red fija, consiste en una malla sostenida por una relinga superior donde se colocan los flotadores y una relinga inferior con pequeños lastres de plomo. La red queda suspendida como una barrera que intercepta a los peces durante su desplazamiento; e) Medio Mundo, es un aro metálico donde va cosida una bolsa cónica de malla fina. Puede estar sujeto a una caña o soga con el fin de desplazarlo por el fondo o a media agua; f) La mojarrera que es una variante de la descrita Caña ya que es una caña delgada y corta con una lineada de nylon que tiene una boya y anzuelo chicos.

Tipo de carnada utilizada: Se detectó el uso de lombriz, mojarra, salame, carne y masa. Esta última es una receta casera que puede tener varios ingredientes, pero básicamente es una pasta que se hace con distintas harinas o maíz fermentado y pisado al que se le agregan condimentos, aromatizantes y colorantes. Sirve para pescar especialmente peces omnívoros como algunas bogas y carpas.

Estación del año en que se pesca: Algunos encuestados hicieron referencia a estaciones y/o meses puntuales del año, otros no especificaron y otros plantearon hacerlo en estaciones consecutivas o no como por ejemplo veranootoño y primavera.

\section{RESULTADOS}

Se realizaron un total de 67 encuestas. Tanto en días de semana como fines de semana, se realizaron 3 muestreos, totalizando 6 días de muestreo. En días de semana se realizaron dos muestreos en otoño y uno en primavera, mientras que en los fines de semana se realizó un muestreo en primavera, otro en verano y otro en otoño. En días de semana se realizaron un total de 19 encuestas mientras que en fin de semana el total fue de 48 . Los datos sociodemográficos tales como edad, localidad, lugar de procedencia de los entrevistados y ocupación se presentan en las Tablas 2 y 3.

El total de especies enunciadas por los encuestados en los distintos escenarios de análisis son 17: cinco pertenecientes al orden Characiformes, nueve a Siluriformes, un Cypriniforme, un Atheriniforme y un Cyprinodontiforme (Tabla 4).

Para todo el período de estudio (Escenario A), las especies mayormente enunciadas son: bagre amarillo ( $P$. maculatus), bagre blanco (P. albicans), mojarras (Astyanax sp.), boga ( $M$. 
obtusidens) y bagre sapo, Rhamdia quelen (Quoy \& Gaimard, 1824) (Figura 3).

Al analizar el consumo de las especies capturadas se observa que el $51 \%$ de las mismas son

Tabla 2 . Datos socio demográficos de los entrevistados. Escenario C: fines de semana.

Table 2. Sociodemographic data of the interviewees. Scenarios C: weekends.

\begin{tabular}{|c|c|c|c|}
\hline Sexo & Edad & Ocupación & Localidad \\
\hline$F$ & 40 & Enfermera & Moreno \\
\hline$M$ & 35 & Herrero & Pilar \\
\hline$M$ & $35-40$ & Empleado & Garín \\
\hline$M$ & 45 & Ferroviario & Escobar \\
\hline $\mathrm{M}$ & 50 & Operario & Campana \\
\hline$M$ & 46 & Empleado de & Escobar \\
\hline$M$ & 32 & No contesta & Escobar \\
\hline$M$ & 31 & Auxiliar de & Escobar \\
\hline M & 35 & Herrero & Pilar \\
\hline $\mathrm{F}$ & 31 & Ama de casa & José C. Paz \\
\hline$M$ & 40 & Médico & Cardales \\
\hline$M$ & 22 & Chofer & Campana \\
\hline$M$ & 40 & Empleado & San Fernando \\
\hline$M$ & 14 & Estudiante & Malvinas \\
\hline $\mathrm{F}$ & 11 & Estudiante & Pablo Nogues \\
\hline$M$ & 33 & Fletero & Tigre \\
\hline M & 12 & Estudiante & Tortuguitas \\
\hline M & 29 & Albañil & Tigre \\
\hline$M$ & 49 & Profesional & Carapachay \\
\hline$F$ & 15 & No contesta & No contesta \\
\hline$M$ & 30 & No contesta & No contesta \\
\hline$M$ & 25 & No contesta & No contesta \\
\hline$M$ & 34 & Empleado & Antes salta \\
\hline$M$ & 33 & Fuerza publica & San miguel \\
\hline$M$ & 50 & Empleado en & $\mathrm{B}^{\circ}$ Las Praderas \\
\hline $\mathrm{F}$ & 33 & Empleada & Campana \\
\hline$M$ & 52 & Terapeuta & Moreno \\
\hline $\mathrm{F}$ & 60 & Empleado & José C. Paz \\
\hline$M$ & 17 & No contesta & Garín \\
\hline M & 18 & No contesta & Garín \\
\hline$M$ & 30 & Comerciante & Pilar \\
\hline$M$ & 26 & Operario & Benavidez \\
\hline$M$ & 33 & Empleado & Avellaneda \\
\hline M & 40 & Empleado & Escobar \\
\hline $\mathrm{F}$ & 39 & Docente & José León Suarez \\
\hline$M$ & 31 & Mozo, cocina & Escobar \\
\hline$M$ & 69 & Jubilado & Pacheco \\
\hline$M$ & 40 & Empleado & Maschwitz \\
\hline$M$ & 70 & Ingeniero & Don Torcuato \\
\hline$M$ & 42 & Albañil & Zarate \\
\hline M & 32 & Operario & JC Paz \\
\hline $\mathrm{F}$ & 46 & Desempleada & Pacheco \\
\hline $\mathrm{F}$ & 41 & Empleada & Maquinista Sabio \\
\hline$M$ & 68 & Jubilado & Campana \\
\hline M & 30 & Empleado & Matheu \\
\hline M & 43 & Cuentapropista & Pacheco \\
\hline$M$ & 36 & Empleado & Escobar \\
\hline$M$ & 57 & Empleado & Córdoba \\
\hline
\end{tabular}

Tabla 3. Datos socio demográficos de los entrevistados. Escenario B: dias de semana.

Table 3. Sociodemographic data of the interviewees. Scenarios B: weekdays.

\begin{tabular}{cclc} 
Sexo & Edad & \multicolumn{1}{c}{ Ocupación } & $\begin{array}{c}\text { Localidad } \\
\text { procedencia }\end{array}$ \\
\hline M & 37 & Albañil & Río Luján \\
M & NC & Albañil & Río Luján \\
M & $30-35$ & Desempleado & Escobar \\
M & $35-40$ & Cloacas/pozos de agua & Escobar \\
M & 20 & Albañil & Otamendi \\
M & 26 & Pozos de agua & Escobar \\
M & $30-35$ & Desempleado & No contesta \\
M & $>30$ & No Contesta & Río Lujan \\
F & 40 & Empleada fabrica & Escobar \\
M & 70 & Jubilado metalúrgico & Río Luján \\
M & 46 & Techista & Campana \\
M & 17 & Albañil & Campana \\
M & 25 & Empleado & Cardales \\
M & 23 & Recolector de residuos & Cardales \\
M & 21 & Albañil & Campana \\
M & 21 & Empleado & Pilar \\
M & 64 & Jubilado & Campana \\
F & 51 & Ama de casa & San Miguel \\
M & 21 & Mecánico & Las Praderas
\end{tabular}

Tabla 4 . Lista de especies enunciadas en las entrevistas. Los números corresponden a la cantidad de veces que los entrevistados enuncian a dicha especie. B: Escenario días de semana, C: Escenario fines de semana.

Table 4. List of species mentioned in the interviews. The numbers correspond to the number of times the interviewees enunciate that species. B: Weekday scenario and C: Weekend scenario.

\begin{tabular}{lllcc} 
Orden & Nombre Científico & Nombre & B & C \\
\hline Cypriniformes & Cyprinus carpio & carpa & 5 & 6 \\
Characiformes & Prochilodus lineatus & sábalo & 4 & 2 \\
Characiformes & Megaleporinus obtusidens & boga & 10 & 15 \\
Characiformes & Hoplias argentinensis & tararira & 2 & 8 \\
Characiformes & Astyanax sp. & mojarra & 5 & 33 \\
Characiformes & Salminus brasiliensis & dorado & 5 & 2 \\
Siluriformes & Iheringichthys labrosus & bagre & 0 & 5 \\
Siluriformes & Luciopimelodus pati & patí & 3 & 4 \\
Siluriformes & Parapimelodus valenciennis & bagre & 5 & 0 \\
Siluriformes & Pseudoplatystoma corruscans & surubí & 1 & 0 \\
Siluriformes & Pimelodella gracilis & quitasueño & 0 & 1 \\
Siluriformes & Pimelodella laticeps & bagre gris & 2 & 1 \\
Siluriformes & Rhamdia quelen & bagre sapo & 8 & 12 \\
Atheriniformes & Odontesthes perugiae & pejerrey & 4 & 2 \\
Cyprinodontiformes & Cnesterodon decemmaculatus & madrecita & 1 & 0 \\
Siluriformes & Pimelodus albicans & bagre & 13 & 16 \\
Siluriformes & Pimelodus maculatus & bagre & 13 & 20
\end{tabular}




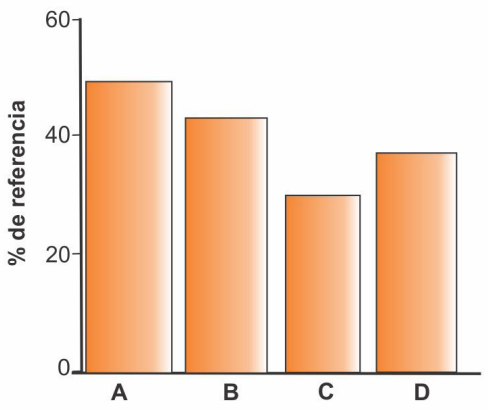

Figura 3. Especies más enunciadas durante todo el periodo de estudio (Escenario A). A: Pimelodus maculatus; B: P. albicans; C: Rhamdia quelen y D: Astyanax sp.

Figure 3. More enunciated species during the entire study period (Scenario A).

consumidas y solo un $6 \%$ no hace referencia al consumo. En cuanto al uso que hacen los entrevistados de los peces, cuando contestan que no lo consumen se observa que el mayor porcentaje corresponde a otros usos (62\%). Se observa que el arte de pesca más utilizado es la caña seguido por el espinel/línea (Figura 4). En cambio, la carnada más utilizada es la lombriz (Figura 5). El mayor porcentaje de encuestados

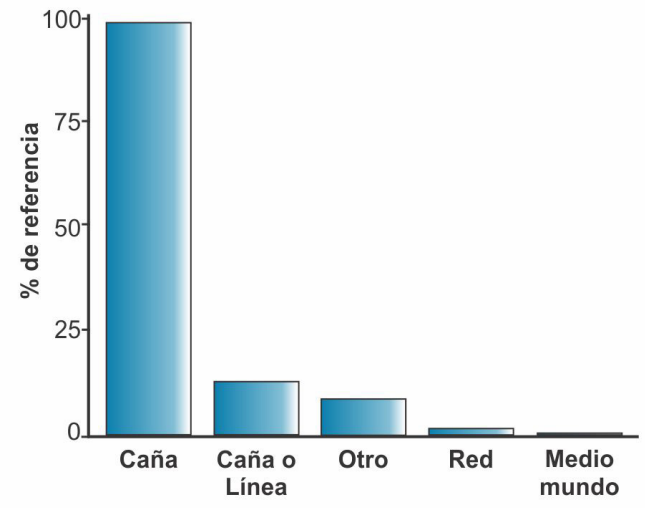

Figura 4. Artes de pesca utilizados durante todo el período de estudio (Escenario A).

Figure 4. Fishing gear used during the entire study period (Scenario A).

respondió que pesca durante todo el año, pero también se registran distintas combinaciones de momentos en los cuales se realiza la actividad (Figura 6).

Días de semana (Escenario B): De las 19 entrevistas realizadas en el $55 \%$ de ellas el lugar de origen de los pescadores fue el partido de Campana, siendo el resto de los partidos

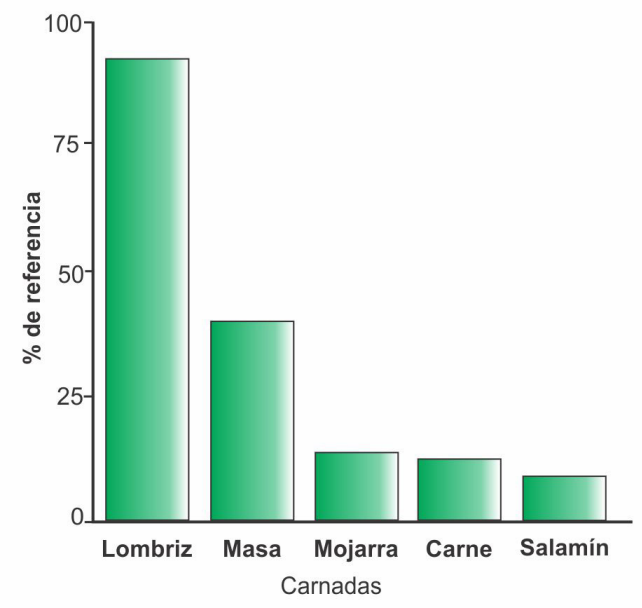

Figura 5. Tipo de carnada utilizada durante todo el período de estudio (Escenario A).

Figure 5. Type of bait used during the entire study period (Scenario A).

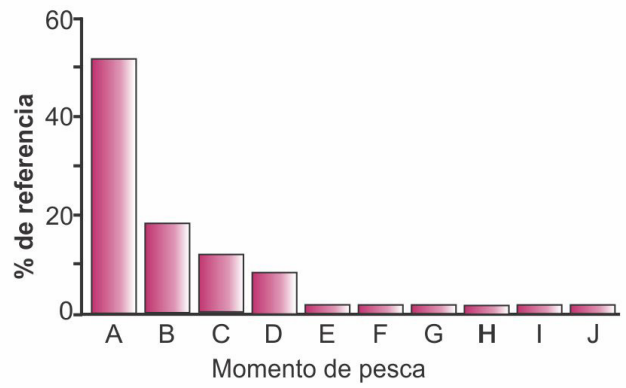

Figura 6. Momento del año elegido para pescar durante todo el período de estudio (Escenario A). A: Todo el año; B: Verano; C: No especifica; D: Primavera / Verano; E: Octubre/Verano; F: Otoño / Primavera; G: Primavera; H: Septiembre / Octubre; I: Septiembre / Verano; J: Verano / Otoño/ Primavera.

Figure 6. Moment of the year chosen to fish during the entire study period (Scenario A). A: All year; B: Summer; C: Does not specify; D: Spring/Summer; E: October / Summer; F: Fall / Spring; G: Spring; H: September / October; I: September / Summer; J: Summer / Fall / Spring.

de Escobar, Maquinista Sabio y de San Miguel (Tabla 2). También es relevante que de los 19 entrevistados el $89 \%$ fueron varones y el $11 \%$ fueron mujeres.

Se identificó un total de 15 especies (Tabla 4) y siendo las especies mayormente enunciadas el bagre amarillo ( $P$. maculatus), el bagre blanco ( $P$. albicans), la boga (M. obtusidens) y el bagre sapo 


\section{(R. quelen) (Figura 7).}

Al analizar el destino de las especies capturadas se observa que el mayor porcentaje de estas (68\%) son consumidas. El uso de los peces que no son para consumo fue mayormente destinado a la venta eventual (83\%).

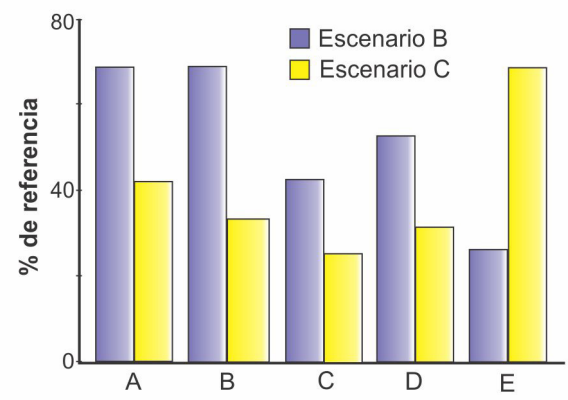

Figura 7. Especies más enunciadas durante el periodo de estudio en los escenarios B (días de la semana) y C (fin de semana). A: Pimelodus maculatus; B: Pimelodus albicans; C: Rhamdia quelen; D: Megaleporinus obtusidens; E: Astyanax sp.

Figure 7. More enunciated species during the study period in scenarios B (weekdays) and C (weekend).

Las ocupaciones de los 13 entrevistados que afirman consumir lo que capturan muestran que dos de ellos son jubilados, tres son empleados y siete poseen oficios de contratación temporarias (plomero, techista y albañil). Solo uno no contestó acerca de su ocupación.

En la Figura 8 puede observarse que el arte de pesca más utilizado fue la caña seguido por el espinel y/o línea. La carnada más utilizada fue la lombriz (Figura 9). El mayor porcentaje de encuestados respondió que pesca todo el año seguido por la época estival (Figura 10).

Fines de semana (Escenario C): De las 48 entrevistas realizadas sólo el 13\% correspondieron a pescadores del partido de Campana, el resto eran de localidades de la Provincia de Buenos Aires que no limitan con el sitio de estudio tales como Zárate, Escobar y Exaltación de la Cruz (88\%). En su mayoría son varones adultos los que van a pescar ( $81 \%)$, pero se observa un porcentaje mayor de mujeres (19\%) durante los fines de semana, dado que el conjunto es más familiar. En la Tabla 2 se consignan las edades de los entrevistados. Del total de especies pescadas, los fines

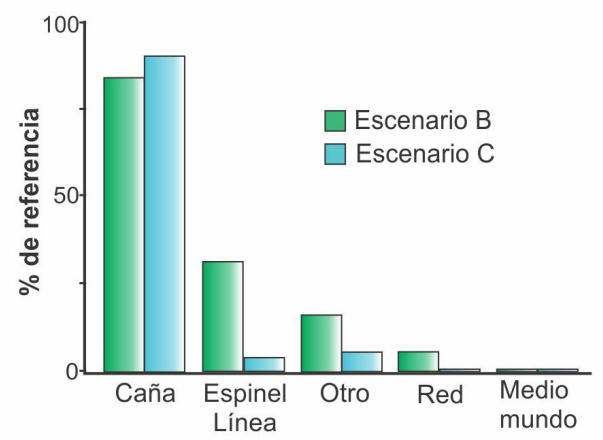

Figura 8. Artes de pesca utilizados durante el periodo de estudio en los escenarios B (días de la semana) y $C$ (fin de semana).

Figure 8. Fishing gear used during the study period in scenarios B (weekdays) and C (weekend).

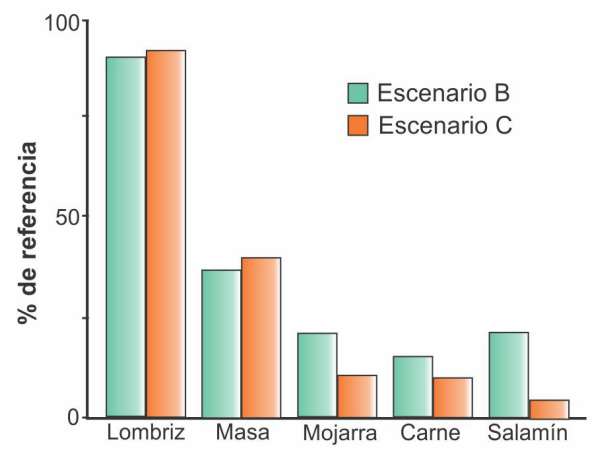

Figura 9. Tipos de carnada utilizadas durante el periodo de estudio en los escenarios B (días de la semana) y $C$ (fin de semana).

Figure 9. Types of baits used during the study period in Scenarios B (weekdays) and C (weekend).

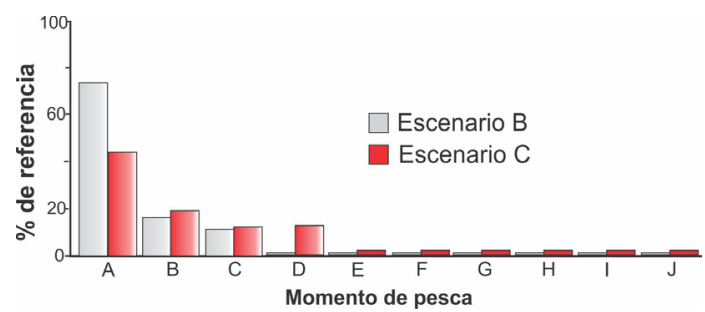

Figura 10. Momento del año elegido para pescar durante el período de estudio en los escenarios $B$ (días de la semana) y C (fin de semana). A: Todo el año, B: Verano; C: No especifica; D: Primavera / Verano; E: Octubre / Verano; F: Otoño / Primavera; G: Primavera; $\mathrm{H}$ : Septiembre / Octubre; I: Septiembre / Verano; J: Verano / Otoño / Primavera.

Figure 10. Moment of the year chosen to fish during the study period in scenarios B (weekdays) and C (weekend). A: All year; B: Summer; C: Does not specify; D: Spring / Summer; E: October / Summer; F: Fall / Spring; G: Spring; H: September / October; I: September / Summer; J: Summer / Fall / Spring. 
de semana sólo 14 son enunciadas por los pescadores. Las especies más enunciadas son: mojarra (Astyanax sp.), bagre amarillo ( $P$. maculatus), boga ( $M$. obtusidens) y bagre sapo (R. quelen) (Figura 7).

Al analizar el destino de las especies capturadas, el $44 \%$ de los entrevistados consume lo que pesca, en cambio $48 \%$ no lo consume y un porcentaje minoritario $(8 \%, N=4)$ no contesta sobre el destino que realiza de las mismas. Las ocupaciones de los 21 entrevistados que afirman consumir lo que capturan, muestran que uno de ellos es jubilado, 12 son empleados en relación de dependencia, dos poseen oficios de contratación temporarias (albañil), una es ama de casa y dos son estudiantes. Por otro lado, de los 23 entrevistados que afirman no consumir lo que capturan se observa que 19 son empleados en relación de dependencia, cuatro poseen oficios de contratación temporaria, un jubilado y un estudiante (Tabla 3).

Del porcentaje de entrevistados que no consume lo que pesca, realizan la actividad de modo deportivo (43\%) y el resto (57 \%) le da otros usos al resultado de la pesca, como por ejemplo alimento de mascotas o lo utiliza como carnada para pescar otras especies.

En este escenario se observa que el arte de pesca más utilizado por los entrevistados para realizar la actividad es la caña, seguidos por otros y el espinel/línea (Figura 8). La carnada más utilizada es la lombriz seguida de la masa, en menor medida utilizan mojarras, carne o salamín (Figura 9). La mayoría de los entrevistados realizan la actividad durante todo el año (Figura 10).

\section{DISCUSIÓN}

Nuestros resultados muestran que las especies más enunciadas a lo largo de todo el estudio fueron: bagre amarillo ( $P$. maculatus), bagre blanco (P. albicans), mojarras (Astyanax sp.), boga ( $M$. obtusidens) y bagre sapo ( $R$. quelen) coincidiendo con lo expresado por Fassel (1997) para esta área.

Los días de semana se enuncian más bagres y bogas que los fines de semana. Esto se debería probablemente a que los pescadores (adultos jóvenes y mayores: 89,5\%), son en su mayoría lugareños que van en busca de peces fundamentalmente para consumo y poseen ocupaciones temporarias y/o son jubilados. Ésto coincide con el arte de pesca más usado que es la caña y el uso de lombrices y masa como carnadas.

Los fines de semana en cambio la especie más enunciada es la mojarra (Astyanax sp.), la cual se obtiene con distintas artes de pesca y carnadas. Ésto posiblemente está relacionado con que, durante el fin de semana, la pesca se realiza en un contexto familiar y deportivo, los rangos etarios están todos representados (adolescentes, adultos, jóvenes y mayores) en su mayoría varones y algunas mujeres. Son, en el caso de los adultos, personas que poseen ocupaciones en relación de dependencia y con lugares de procedencia $(87,5 \%)$ que no son los partidos que limitan con el sitio de análisis. Estos resultados permiten afirmar que en el área de estudio se realiza pesca de subsistencia los días de semana mientras que los fines de semana la pesca tiene un valor recreativo y de características familiares.

En cuanto al consumo nuestros resultados totales permiten plantear que el porcentaje de consumo es mayor que el de no consumo. Al relacionarlo con los escenarios de días de semana y fin de semana se observa que el mayor consumo se registra durante días de semana (68\%) en contraste con el fin de semana es $44 \%$. El porcentaje de no consumo muestra en los resultados totales que el mismo se debe a un uso distinto al deportivo, que corresponde a nuestra categoría de "Otros usos" (carnada o alimento de mascotas - 62\%). Al relacionar esta categoría de "No uso" se observa que en días de semana (83\%) es mayor que en fines de semana (57\%). Creemos que está relacionado con que en los días de semana la pesca es de subsistencia y lo que se pesca y no se consume es para la venta (carnada) o alimento de mascotas. En cambio, los fines de semana, la pesca es deportiva y/o le dan otro uso.

La pesca a lo largo de todo el año en la cuenca inferior del río Luján y los usos que realizan los pobladores de esta actividad nos permite ampliar el conocimiento actual de su fauna íctica. Esto es fundamental para proteger y conservar la biodiversidad presente en la 
cuenca, así como también realizar sugerencias sobre los usos del territorio. En este contexto los resultados obtenidos en este estudio permiten plantear la importancia que tiene este territorio sobre la ribera del río Luján para los pobladores de la zona y de las regiones cercanas dado que es un sitio que ofrece un espacio para realizar tanto pesca de subsistencia los días de semana y como un espacio de esparcimiento familiar los fines de semana.

\section{AGRADECIMIENTOS}

Queremos agradecer por la colaboración en la toma de datos en terreno en al menos uno de los muestreos a Diana Cruz, Florencia Marotta, Nahuel Marchisio y Roberto Fabián Bó. Agradecer además a Miguel Falcón por su colaboración en la toma de datos en terreno y la logística de trabajo en el terreno. A la Reserva Natural Otamendi de la Administración de Parques Nacionales por facilitar la participación en la implementación y análisis de las encuestas de la Lic. Liliana Ciotek (Área de conservación). Este trabajo fue financiado con fondos del programa UBANEX 7 y UBANEX 8 de la Universidad de Buenos Aires, (directora Paula Courtalon).

\section{REFERENCIAS}

Abba, M. \& Cassini, M. (2010). A comparison of two methods for acquiring ecological data on armadillos from Argentinean pampas: field work vs interviews. Interciencia, 35: 450-454.

Almirón, A., Casciotta, J., Ciotek, L. y Giorgis, P. (2015). Guía de los Peces del Parque Nacional Pre-Delta. 2da ed. Ciudad Autónoma de Buenos Aires: Administración de Parques Nacionales.

Andrade, M. I. (1986). Factores del deterioro ambiental de la cuenca del Río Luján. Serie Contribución del Instituto de Geografía, Facultad de Filosofía y Letras, Universidad de Buenos Aires. Recuperado en http://ri.unlu.edu. ar/xmlui/handle/rediunlu/376 (fecha de acceso 26/07/2019)

Arias Arias, P. (comp.). (1988). Artes y métodos de pesca en aguas continentales de América Latina.
COPESCAL Documento Ocasional, (4): 178 p.

Bonetto, A. A., Neiff, J. J. \& Di Persia, D. H. (1986). The Paraná River system. In: Davies B.R., Walker K.F. (Eds.). The ecology of river systems (pp. 541-598). Monographiae Biologicae, vol 60. Dordrecht: Springer.

Brea, J. D., Reyna, J. y Spalletti, P. (2007). Diagnóstico del funcionamiento hidrológico hidráulico de la cuenca del río Luján, Provincia de Buenos Aires. Delimitación de las zonas bajo riesgo hídrico. Laboratorio de Hidráulica Aplicada, Instituto Nacional del Agua, Argentina.

Buzai, G. y Principi, N. (2017). Identificación de áreas de potencial conflicto entre usos del suelo en la cuenca del río Luján, Argentina. Revista Geográfica de América Central, 3(59): 91-124.

Ciotek, L., Giorgis, P., Almirón A. y Casciotta, J. (2017). Relevamiento ictiológico de la Reserva Natural Otamendi - APN, Argentina. Informe APN. V Simposio Argentino de Ictiología. Corrientes, Argentina.

Cruz, D. y Courtalon, P. (2017). Percepciones y referencias acerca del uso de fauna por la comunidad aledaña a la Reserva Natural Otamendi, Campana, Argentina. Ecología Austral, 27: 242-251.

Fassel, J. (1997). Río contaminado atraviesa Reserva 4,Natural Estricta Otamendi. El Mensajero, semanario independiente, 12 (316): 4.

Goldberg, S., Cirera, I., Parella, M., Benítez, A. Bulos, L. y Troncoso, A. (1995). Caracterización climática y agroclimática de la cuenca del Río Luján. En: Resúmenes I Jornadas sobre la cuenca del río Luján.

Hales J, \& Petry P. (2015). Freshwater ecoregions of the world (FEOW). 345: Lower Paraná. Recuperado en: http://www.feow.org/ ecoregions/details/345 (fecha de acceso: 13/08/2019),

Kandus, P., Quintana, R. D., y Bó, R. F. (2006). Patrones de paisaje y biodiversidad del Bajo Delta del Río Paraná. Buenos Aires: Pablo Casa Mayor Ediciones. 
Liotta, J., Wagner, M. y Giacosa, B. (2003). Peces de la Reserva Natural Otamendi. En: Haene, E. y Pereira, J. (Ed.). Fauna de Otamendi. Inventario de los animales vertebrados de la Reserva Natural Otamendi (pp. 17-27). Buenos Aires: Monografía de Aves Argentinas, Temas de Naturaleza \& Conservación.

Lucca Irizarry, N y Berríos Rivera, R. (2009). Investigación cualitativa: Fundamentos, diseños y estrategias. Puerto Rico: Ediciones SM.

Monroy-Vilchis, O., Cabrera, L., Suárez, P., ZarcoGonzález, M. M., Rodríguez-Soto, C., y Urios, V. (2008). Uso tradicional de vertebrados silvestres en la Sierra Nanchititla, México. Interciencia, 33: 308-313.

Morello, J., Buzai, G., Baxendale, C., Matteucci, S., Rodríguez, A., Godagnone, R. y Casas, R. (2000). Urbanización y consumo de tierra fértil. Ciencia Hoy, 10(55): 50-61.

Navarro, M. (2005). Conocimientos y percepciones sobre la fauna por los habitantes de la Isla Cozumel. Tesis profesional para obtener el título de Licenciado en Biología, Centro Universitario de Ciencias Biológicas y Agropecuarias, Universidad de Guadalajara.

Padín, O. H. e Iriart, N. R. (1991). Artes y métodos de pesca utilizados en la provincia de Buenos Aires. En: López, H. y Tonni, E. (Coords.). Situación
Ambiental de la Provincia de Buenos Aires. A. Recursos y rasgos naturales en la evaluación ambiental (pp. 5-31). La Plata: Comisión de Investigaciones Científicas de la Provincia de Buenos Aires (CICBA)

Plataroti, M. C. (2010). Caracterización de la calidad del agua de una sección del río Luján: Efectos sobre el fitoplancton. Tesis de licenciatura, Facultad de Ciencias Exactas y Naturales, UBA.

Tejada, R., Chao, E., Gómez, H., Painter, R. E. L. y Wallace, R. B. (2006). Evaluación sobre el uso de la fauna silvestre en la Tierra Comunitaria de Origen Tacana, Bolivia. Ecología en Bolivia, 41:138-148.

Viglizzo, E. F., Frank, F. C. y Carreño, L. (2005). Situación ambiental en las ecorregiones Pampa y Campos y Malezales. En: Brown, A., Martínez Ortiz, U., Acerbi, M. y Corcuera, J. (Eds.). La situación ambiental argentina (pp. 263 269). Buenos Aires: Fundación Vida Silvestre Argentina.

Vodouhê, F., Coulibaly, O., Adégbidi, A. \& Sinsin, B. (2010). Community perception of biodiversity conservation within protected areas in Benin. Forest Policy and Economics, 12: 505-512. 